\title{
A simple anesthetic technique to eliminate pain and optimize patient satisfaction for chalazion incision and curettage
}

\begin{abstract}
Purpose: To develop a painless anesthetic technique for chalazion incision and curettage in order to optimize patient comfort and satisfaction.

Method: Four patients with upper lid chalazion refractive to conservative treatment were scheduled for incision and curettage. The technique involved the application of eutectic lidocaine $2.5 \%$ and prilocaine $2.5 \%$ (EMLA) prior to subcutaneous injections of chloroprocaine (Nesacaine) and $2 \%$ lidocaine. Further anesthesia of the conjunctiva was provided with a tetracaine soaked cotton applicator for 30 seconds prior to $2 \%$ subconjunctival injection adjacent to the tarsal plate. Pain was assessed with numerical score of zero to ten prior and during the procedure. Overall patient satisfaction was evaluated using a Likert satisfaction scale from 1-5.
\end{abstract}

Results: All patients documented zero pain with the anesthetic process as well as during the procedure. Also, patient satisfaction received the highest rating, 5, for each patient.

Conclusion: Minimizing discomfort is important as patients with a low threshold of pain can increase the risk of globe injury due to poor patient cooperation during injection. Thus a technique which alleviates pain-related anxiety may aid in minimizing unnecessary complications and improving overall satisfaction.

Keywords: chalazion, painless, patient satisfaction
Volume 7 Issue 7 - 2017

\author{
Thomas D LoBue,' Richard A Giovane, ${ }^{2}$ \\ Nicholas Bahl, ${ }^{3}$ Erin Schaefer ${ }^{\prime}$ \\ 'LoBue Laser \& Eye Medical Centers, USA \\ ${ }^{2}$ Department of Family Medicine, University of Alabama, USA \\ ${ }^{3}$ Department of General Surgery, Texas Tech University Health \\ Sciences Center at the Permian Basin, USA
}

Correspondence: Thomas D LoBue, Medical Director and CEO at LoBue Laser and Eye Medical Center Inc. 40700 California Oaks Rd, Ste 106, Murrieta, CA 92562, USA, Tel 95 I-696-I I35, Email Thomas.lobue@lobueeyecenters.com

Received: December 12, 2017 | Published: December 21, 2017

\section{Introduction}

A chalazion is a localized area of granulomatous inflammation associated with lipid deposits and swelling of the eyelid. It occurs due to the obstruction of sebaceous glands, meibomian or Zeis, of which the former is more common. The meibomian gland may be obstructed due to varying etiologies including infection, inflammation, or neoplastic lesions. Chalazia may have a slow, insidious onset with minimal symptoms. Most commonly, localized inflammation occurs in the upper lid, with an immobile, hard, mass in the tarsal plate. If large enough, an upper lid chalazion can induce visual disturbances by creating mechanical ptosis or causing increased corneal astigmatism. The chalazion may produce pain if it grows large enough to cause distention of the terminal branches of the ophthalmic (V1) or maxillary (V2) sensory nerve endings. Terminal branches of the ophthalmic division supply the upper eyelid as the lacrimal, supraorbital, and supratrochlear nerves. Terminal branches of the maxillary division supply the lower eyelid as the zygomaticofacial and infraorbital nerves. In addition, sensation of both upper and lower lids results from innervations of the infratrochlear nerve.

Although chalazia may regress spontaneously without treatment in up to 25 to $43 \%$ of patients, ${ }^{2}$ a majority require intervention. First line management includes conservative treatment (eg, hot compress and lid hygiene) with surgical options of incision and curettage (I\&C) or intralesional or extralesional steroid injections for refractory cases. However, most ophthalmologists perform Incision and curettage after a trial of conservative management has failed. ${ }^{3}$ Incision and curettage involves topical proparacaine and infiltrated anesthetic. Additionally, the injection of local anesthetic solutions is often very uncomfortable for most patients. ${ }^{4}$ The discomfort is largely associated with pain and burning sensation from the infiltration of skin and subcutaneous tissues with a local anesthetic. ${ }^{5,6}$ Often it is severe enough to be the most unpleasant part of a minor surgical procedure. Surgeons have attempted to address this discomfort by using an additional topical anesthetic such as $2 \%$ lidocaine gel ${ }^{7}$ or a mixture of eutectic lidocaine $2.5 \%$ and prilocaine $2.5 \%$ (EMLA). ${ }^{8}$ However, complete resolution of pain was not attained ${ }^{7,9}$ or the method was cumbersome in most clinical settings. Therefore, we derived a simple, quick technique to eliminate any discomfort associated with anesthetic injection or incision in Chalazion removal. We believe this technique will aid in improving patient pain and overall satisfaction regarding surgical management of chalazion treatment.

\section{Methods}

Informed consent was obtained from 4 consecutive patients undergoing incision and curettage for chalazion removal. All patients were over 18 years of age. Subjects with previous chalazion or lid surgery were excluded. Also, patients who had systemic conditions which involved the habitual use of analgesics were not included in the case series. Pain was assessed with a numerical score of ranging from zero to ten prior and during the procedure. Overall patient satisfaction was also evaluated with a Likert satisfaction scale ranging from 1-5. One to Five was represented as very poor, poor, fair, good, and excellent. 


\section{Anesthetic technique}

A mixture of eutectic lidocaine $2.5 \%$ and prilocaine $2.5 \%$ (EMLA) is placed on the chalazion for 10 minutes (Figure 1). After 10 minutes EMLA cream is removed with a Q tip (Figure 2). The chalazion is marked with a sterile skin marker (Figure 3). Next a subcutaneous injection near the chalazion is performed with chloroprocaine $\mathrm{HCl}$ (Nesacaine) on a TB syringe with a 27-gauge needle (Figure 4). Lateral to the previous anesthetic, $2 \%$ lidocaine is inserted subcutaneously with a 27-gauge needle (Figure 5). The lid is draped and then prepped with Povidone-iodine (Betadine) (Figure 6). The chalazion is isolated with the chalazion clamp with the lip everted (Figure 7). The superior aspect of the tarsal plate and the adjacent conjunctiva are anesthetized with a cotton-tipped applicator soaked in tetracaine for about 30 seconds (Figure 8). The conjunctiva adjacent to the tarsal plate is injected with $2 \%$ lidocaine subconjunctivally. Ballooning of the conjunctiva can be witnessed during anesthetic infiltration (Figure 9). Finally, routine incision and curettage of the chalazion is performed.

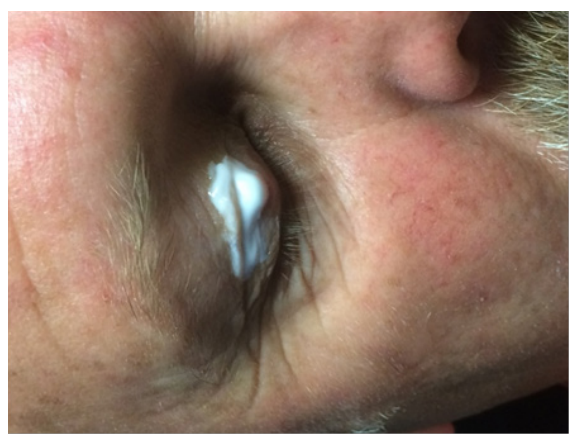

Figure I A mixture of eutectic lidocaine $2.5 \%$ and prilocaine $2.5 \%$ (EMLA) is placed on the chalazion for 10 minutes.

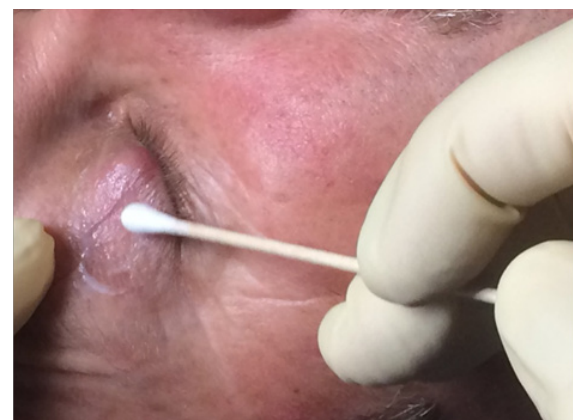

Figure 2 After 10 minutes EMLA cream is removed with a $\mathrm{Q}$ tip. Chalazion is seen nasal to the $\mathrm{Q}$ tip.

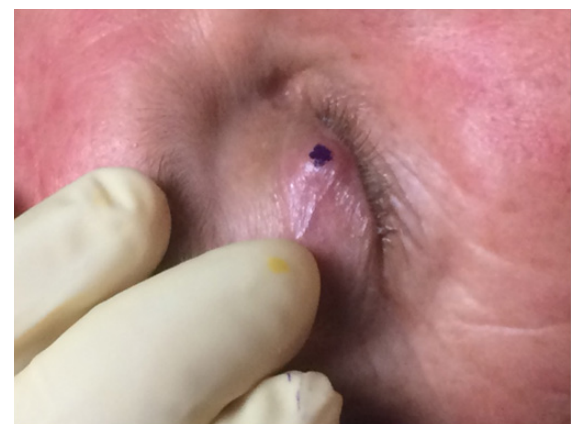

Figure $\mathbf{3}$ The chalazion is marked with a sterile skin marker.

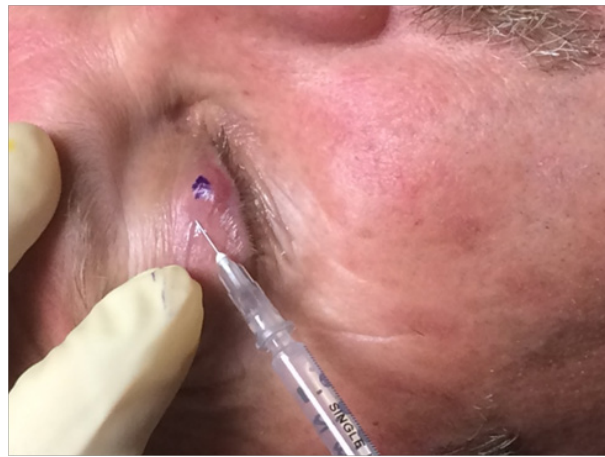

Figure 4 Injecting the chalazion subcutaneously with chloroprocaine $\mathrm{HCl}$ (Nesacaine) on a TB syringe with a 27 guage needle.

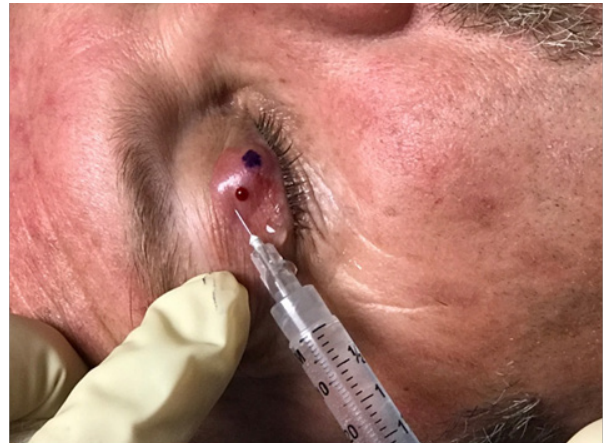

Figure 5 Temporal to previous anesthetic, 2\% lidocaine is inserted subcutaneously with a 27 -gauge needle.

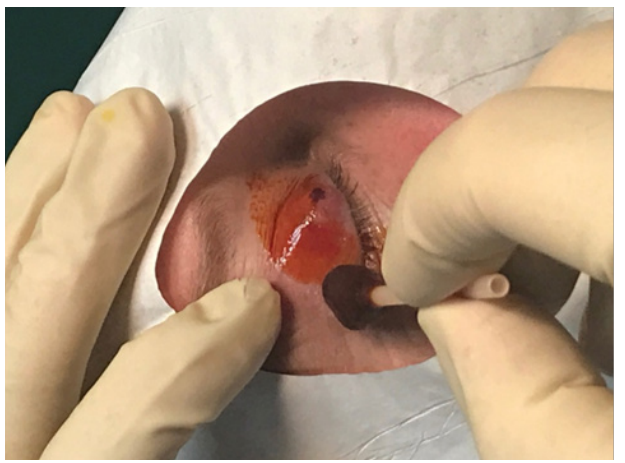

Figure 6 The lid is drapped and then prepped with Povidone-iodine (betadine).

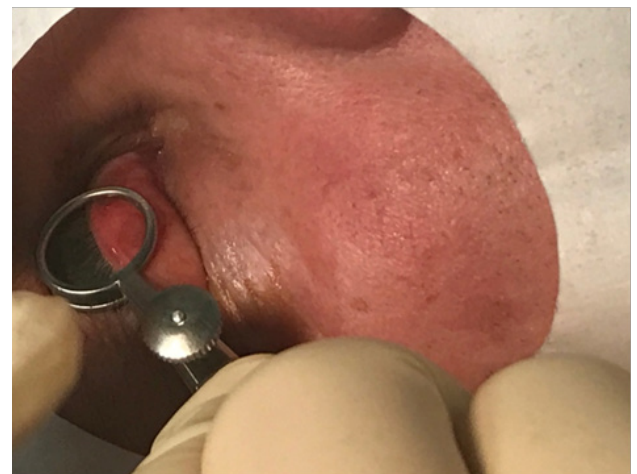

Figure 7 The chalazion is isolated with the chalazion clamp with the lid everted. 


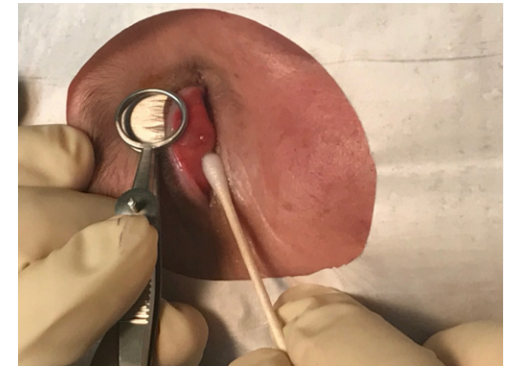

Figure 8 The superior aspect of the tarsal plate and the adjacent conjunctiva are anesthetized with a cotton tipped applicator soaked in tetracaine for about 30 seconds.

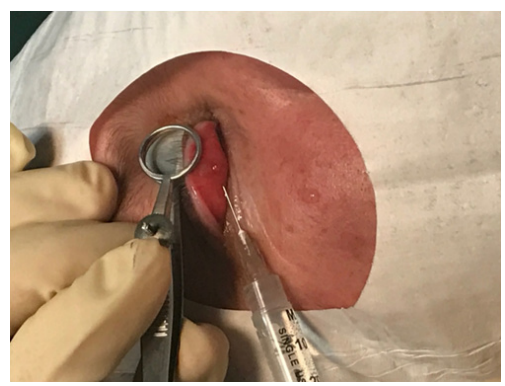

Figure 9 The conjunctiva adjacent to the tarsal plate is injected with $2 \%$ lidocaine subconjunctivally. Ballooning of the conjunctiva can be witnessed during anesthetic infiltration.

\section{Results}

All patients documented zero pain with the anesthetic process as well as during the procedure. Also, patient satisfaction received the highest rating, excellent, by each patient. The technique has been performed for the past several years with similar anecdotal results.

\section{Discussion}

Minimizing pain is relevant for the surgeon as patients with a low pain threshold can increase the risk of globe injury due to poor patient cooperation during injection. ${ }^{10}$ Thus a technique which alleviates pain-related anxiety may aid in minimizing unnecessary complications or risks for the patient. Typically the routine incision and curettage procedure for chalazion removal results in a significant pain scale. Goawalla et al. ${ }^{11}$ documented a median pain score of $7 / 10$ for 45 patients treated by I\&C when using only subcutaneous injection of $2 \%$ lidocaine with a drop of $0.5 \%$ proxymethocaine. Buik et al. ${ }^{12}$ demonstrated a median pale scale of $6 / 10$ for 15 patients using only local injection of $2 \%$ lidocaine with $1: 10,000$ adrenaline subcutaneously.

Nevertheless, a common alternative surgical procedure for chalazion removal, which has documented less pain, ${ }^{11,12}$ is intralesional or extralesional steroid injection. Complete absence of pain was documented when combing topical cutaneous anesthetic such as EMLA prior to transcutaneous injection of triamcinolone acetonide (TA) ]. ${ }^{12}$ Although transcutaneous injection using EMLA resulted in no pain, transcutaneous steroid administration is associated with higher reports of depigmentation at the injection site, globe perforation, and anterior segment ischemia. ${ }^{3,11}$ As a result, a transconjunctival approach is more commonly performed. Using this approach, Goawalla et al. ${ }^{11}$ documented comparable pain score to $\mathrm{I} \& \mathrm{C}$ with a median score of
$6 / 10$ for 50 patients. However, the conjunctiva was solely anesthetized with a drop of $0.5 \%$ proxymethocaine.

Our technique focuses on cutaneous as well as conjunctival anesthesia prior to infiltrative anesthetic injection. A common topical anesthetic which has been successfully used for transcutaneous injection is EMLA. It has been shown to provide $5 \mathrm{~mm}$ of transcutaneous anesthesia after 2hours of application. ${ }^{13}$ Thus the depth is sufficient for areas of the face. Wessels et al. ${ }^{8}$ documented that EMLA applied to the lid for one hour resulted in painless subcutaneous injection in pediatric patients. However, we noted the same results in only 10 minutes of EMLA application on the affected area. Thus in settings when time constraints are prevalent, our results support that 10 minutes of EMLA application may be more than sufficient to eliminate pain from anesthetic injection. We have noted that some patients are not completely anesthetized solely with subcutaneous anesthesia. Thus an additional subconjunctival injection may be necessary to completely eliminate pain during the procedure. However, EMLA cannot be used on the palpebral conjunctiva where the main incision is created. As a result, we use anesthetic-soaked applicators (eg., tetracaine) to quickly numb the conjunctiva prior to injection. Other types of anesthetics have been suggested such as $2 \%$ lidocaine gel. However, $2 \%$ lidocaine gel was not sufficient alone to prevent pain associated with I\&D procedure. ${ }^{7}$ Although our sample size is very small. The technique has been performed for over ten years with similar anecdotal results. Overall our technique greatly minimizes the pain and discomfort associated with an inoffice chalazion removal. It is ideal for patients who have mild to moderate anxiety due to a low threshold of pain. It can be applied to most consenting adults, in order optimize patient satisfaction and cooperation. However, the technique also has potential to be applied in a pediatric setting where chalazion is common as well. Reducing pain could improve cooperation and potentially decrease the amount of patients requiring general anesthesia.

\section{Conclusion}

In summary, we have demonstrated a technique for painless anesthesia and incision of a chalazion. Our technique involves a 10 -minute application of eutectic lidocaine $2.5 \%$ and prilocaine $2.5 \%$ (EMLA) prior to subcutaneous injection of chloroprocaine (Nesacaine) and 2\% lidocaine. Further anesthesia of the conjunctiva is provided with a tetracaine soaked cotton applicator for 30 seconds prior to $2 \%$ subconjunctival injection adjacent to the tarsal plate. Overall, minimizing discomfort is important as patients with a low threshold of pain can increase the risk of globe injury due to poor patient cooperation during injection. Thus a technique which alleviates pain-related anxiety may aid in minimizing unnecessary complications.

\section{Acknowledgments}

None.

\section{Conflicts of interest}

The authors did not report any potential conflict of interests.

\section{Funding}

None. 


\section{References}

1. Jin KW, Shin YJ, Hyon JY. Effects of chalazia on corneal astigmatism Large-sized chalazia in middle upper eyelids compress the cornea and induce the corneal astigmatism. BMC Ophthalmol. 2017;17(1):36.

2. Cottrell DG, Bosanquet RC, Fawcett IM. Chalazions: the frequency of spontaneous resolution. Br Med J (Clin Res Ed). 1983;287(6405):1595.

3. Ahmad S, Baig MA, Khan MA, et al. Intralesional corticosteroid injection vs surgical treatment of chalazia in pigmented patients. $J$ Coll Physicians Surg Pak. 2006;16(1):42-44.

4. Bell RW, Butt ZA, Gardner RF. Warming lignocaine reduces the pain of injection during local anaesthetic eyelid surgery. Eye (Lond). 1996;10( Pt 5):558-560.

5. Morris RW, Whish DK. A controlled trial of pain on skin infiltration with local anaesthetics. Anaesth Intensive Care. 1984;12(2): 113-114.

6. Morris R, McKay W, Mushlin P. Comparison of pain associated with intradermal and subcutaneous infiltration with various local anesthetic solutions. Anesth Analg. 1987;66(11):1180-1182.

7. Li RT, Lai JS, Ng JS, et al. Efficacy of lignocaine $2 \%$ gel in chalazion surgery. Br J Ophthalmol. 2003;87(2):157-159.
8. Wessels IF, Wessels GF. Lidocaine-prilocaine cream for local-anesthesia chalazion incision in children. Ophthalmic Surg Lasers. 1996;27(6):431433.

9. Osayande OO, Mahmoud AO, Bolaji BO. Comparison of topical lidocaine $[2 \%$ gel] and injectable lidocaine [2\% solution] for incision and curettage of chalazion in Ilorin, Nigeria. Niger Postgrad Med J. 2010;17(4):270-276.

10. Hay A, Flynn HW, Hoffman JI, et al. Needle penetration of the globe during retrobulbar and peribulbar injections. Ophthalmology. 1991;98(7):1017-1024.

11. Goawalla A, Lee V. A prospective randomized treatment study comparing three treatment options for chalazia: triamcinolone acetonide injections, incision and curettage and treatment with hot compresses. Clin Exp Ophthalmol. 2007;35(8):706-712.

12. Biuk D, Matic S, Barac J, et al. Chalazion management--surgical treatment versus triamcinolon application. Coll Antropol. 2013;37(Suppl 1):247-250.

13. Bjerring $\mathrm{P}$, Arendt-Nielsen L. Depth and duration of skin analgesia to needle insertion after topical application of EMLA cream. Br J Anaesth. 1990;64(2):173-177. 